\title{
Análisis bibliométrico del turismo deportivo y los medios sociales
}

\section{Bibliometric analysis of sports tourism and social media}

CARMEN SARAH EINSLE

Universidad Nacional de Educación a Distancia (UNED), Madrid. España carmen.einsle@gmail.com

ORCID: https://orcid.org/0000-0002-9829-7193

\section{GREGORIO ESCALERA IZQUIERDO}

Universidad Nacional de Educación a Distancia (UNED), Madrid. España gescalera@cee.uned.es

Recibido: 21-05-2021. Aceptado: 21-07-2021.

Cómo citar / Citation: Einsle, C.S. y Escalera, G. (2021). Análisis bibliométrico del turismo deportivo y los medios sociales. Ágora para la Educación Física y el Deporte, 23, 308-327.

DOI: https://doi.org/10.24197/aefd.0.2021.308-327

Resumen. El turismo deportivo, aunque siendo un tipo de turismo relativamente nuevo, está experimentando un gran crecimiento en todas sus vertientes. Por otro lado, la evolución digital está en auge continuo, igual que el impacto generado por los medios sociales. De la consideración del incremento en ambos sectores surge la necesidad de examinar cómo la investigación científica se ha adaptado a estas nuevas realidades. Como consecuencia, el objetivo del presente estudio es mostrar el estado actual de las investigaciones en el turismo deportivo y los medios de comunicación sociales. Para ello se llevó a cabo una búsqueda en las bases de datos WoS y Scopus. Se aplicaron métodos cuantitativos para el análisis de co-palabras mediante el uso de la herramienta SciMat. Los resultados obtenidos ponen de manifiesto que los principales temas motores de los tres periodos analizados, "sport", "human" y "sport-event", se encuentran estrechamente relacionados con "social-media" y "social-network".

Palabras clave. Análisis bibliométrico; turismo deportivo; evento deportivo; medios sociales; mapeo científico

Abstract. Sports tourism, although being a relatively recent type of tourism, has grown significantly in all its aspects. The digital evolution itself is in a constant growth, as well as the generated impact of social media. Considering the increase in both sectors, an examination of how scientific research has adapted to these new realities is required. Hence, the aim of this study is to show the current state of scientific research regarding the field of sports tourism and social media. For this purpose, a search in the databases of WoS and Scopus was performed. 
Quantitative methods were applied for a co-word analysis, using the SciMat tool. The results conclude, that the main motor themes of the three analysed periods, "sport", "human" and "sport-event", are closely related to "social-media" and "social-network".

Keywords. Bibliometric analysis; sports tourism; sport events; social media; science mapping

\section{INTRODUCCIÓN}

Los conceptos turismo y deporte se están relacionando desde hace varias décadas, aunque los primeros estudios se enfocaron más en las vacaciones deportivas que en el turismo deportivo (De Knop, 1990; Glyptis, 1982). No fue hasta los años 90 que se comenzó a relacionar los dos conceptos y a ver el turismo deportivo como un sector que necesitaba una denominación propia (Gammon y Robinson, 2003; Gibson, 1998; Weed, 2005).

En general, se puede dividir el turismo deportivo en activo y pasivo. Independientemente de si se trata de turistas participantes en deportes o turistas pasivos, que asisten como espectadores a eventos deportivos, el turismo deportivo puede estar clasificado como aquel viaje fuera del entorno habitual para participar o asistir a un evento deportivo o en un tipo de deporte recreacional siendo la motivación principal del viaje el deporte (Gammon y Robinson, 2003).

Para Weed y Bull (2009), el turismo deportivo es "un fenómeno social, económico y cultural que surge de la interacción única de la actividad, las personas y el lugar" (p.258), debido a que manifiestan ya con anterioridad que el turismo deportivo está interrelacionado pero que supone más que la sola suma entre deporte y turismo (Weed, 2005).

El turismo es una de las industrias que contribuyen en mayor medida a la economía global. Dentro de ello, el turismo deportivo es uno de los sectores turísticos con más crecimiento (World Tourism Organization, s.f.). Así, por ejemplo, 180 millones de personas viajaron en el año 2019 para atender de manera activa o pasiva a un evento deportivo en los Estados Unidos, lo cual supone un incremento de 5,9\% desde el año 2015. Los turistas deportivos, junto con las organizaciones y los destinos de los eventos gastaron 45,1 mil millones de US\$, un aumento del 16,7\% en el periodo de los cinco años entre el 2015 y el 2019. (Tourism Economics, 2020). 
Estudios en el ámbito deportivo relacionados con los medios sociales, se han centrado en la motivación de los aficionados para interactuar en los medios sociales (Stavros, Meng, Westberg, \& Farrelly, 2014), en las percepciones de los atletas del beneficio del uso del social media durante grandes eventos deportivos (Hayes, Filo, Riot, \& Geurin, 2019) y en la gestión del deporte mediante el branding y la interacción con los aficionados (Anagnostopoulos, Parganas, Chadwick, \& Fenton, 2018).

Los medios sociales no son un fenómeno reciente y tienen sus orígenes aún antes de la llegada de la denominada web 2.0. Aun así, es a partir de la aparición de la web 2.0 que se desarrollan los medios sociales, ya que desde entonces es posible la comunicación bidireccional de contenido, distintivo de los medios sociales (Kaplan \& Haenlein, 2010). La interacción y el engagement son elementos clave en el marketing relacional (Filo, Lock, \& Karg, 2015; Grönroos, 2004). La posibilidad de interacción de los atletas, los aficionados y las organizaciones en las redes sociales es un factor importante que considerar en las estrategias de marketing. Sin embargo, en muchas organizaciones deportivas las estrategias del social media marketing no están lo suficientemente definidas para aprovecharse de todas las oportunidades que brinda la comunicación bidireccional en estos medios (Girginov et al., 2009; González, 2012).

Aunque traen múltiples beneficios y oportunidades, no hay que obviar los retos y efectos negativos que puede conllevar el uso de los medios sociales tanto para el usuario como para las organizaciones (Abeza, O'Reilly, \& Reid, 2013; Baccarella, Wagner, Kietzmann, \& McCarthy, 2018).

Los cuatros motivos que presentan los aficionados cuando conectan con su equipo por medios sociales son: pasión, esperanza, aprecio y compañerismo (Stavros et al., 2014). El deporte se caracteriza por altos niveles de conexión emocional e identificación y esto lo diferencia de otras actividades de ocio (Sutton, McDonald, Milne, \& Cimperman, 1997). Los medios sociales ofrecen la posibilidad de mejorar esta conexión emocional y el compromiso de los aficionados mediante la oferta de experiencias nuevas e interactivas (Meng, Stavros, \& Westberg, 2015).

Aunque como se ha podido observar, los estudios en el ámbito de los medios sociales están en auge, en el sector de la gestión deportiva las investigaciones aún son incipientes (Meng et al., 2015). A pesar de la 
existencia de poca investigación sobre el campo de los medios sociales en el ámbito de la gestión deportiva, es una temática con un interés académico progresivo y que presenta oportunidades para la conexión de estos dos campos (Filo et al., 2015; López-Carril, Villamón, \& Sanz, 2019).

Un análisis más empírico de la relación de los medios sociales online con el sector del turismo deportivo es necesario, considerando que tanto en el sector turístico deportivo, como en el social media se están efectuando grandes incrementos. El uso de las redes sociales en el turismo deportivo es un fenómeno reciente, por lo tanto, el objetivo del presente estudio fue examinar y mostrar el estado actual de la investigación científica en este campo mediante el uso de técnicas bibliométricas.

\section{Metodología}

Las revisiones de la literatura juegan un rol importante para examinar el estado actual de los conocimientos en un determinado campo y para orientar las investigaciones futuras (Cropanzano, 2009). Para que esta revisión tenga valor científico, debe ser sistemática y regirse por algunos parámetros (Kitchenham, 2004).

Con el fin de obtener la visión del estado actual del campo más precisa, se optó por la aplicación de métodos estadísticos a la presente revisión. Pritchard (1969) fue el primero en establecer el término "bibliométrica" para evitar el uso del término confuso "bibliografía estadística" y referirse de tal manera a la aplicación de métodos matemáticos y estadísticos a documentos. La bibliometría consta de dos aspectos principales, el análisis de rendimiento y el mapeo científico (Noyons, Moed, \& Luwel, 1999). Mediante el mapeo científico se representa la estructura cognitiva de un campo de investigación (Cobo, López-Herrera, Herrera-Viedma, \& Herrera, 2011). Para esta representación se usan diferentes técnicas, siendo las más frecuentemente usadas, la co-citación de documentos (Small, 1973) y el análisis de copalabras (Callon, Courtial, \& Laville, 1991).

Para llevar a cabo el presente estudio se realizaron búsquedas en las dos principales bases de datos a nivel global de referencias bibliográficas y citas de publicaciones periódicas, la Web of Sciences (WOS) y Scopus. WOS, de la empresa Clarivate Analytics, que ofrecen una colección de bases de datos de diferentes disciplinas con una cobertura de información 
desde 1900 hasta la actualidad. Scopus, lanzada por la empresa Elsevier en el año 2004 es una base de datos multidisciplinar que ofrece literatura revisada por pares (Fundación Española para la Ciencia y la Tecnología, s.f.).

Con respecto a las palabras clave se realizó la búsqueda avanzada en las bases de datos anteriormente mencionados con los siguientes términos: "sport", "tourism", "event" y "social media". Se utilizaron los operadores booleanos "AND" y "OR" para especificar y ampliar la búsqueda. Además, para generalizar la búsqueda se implementó el símbolo "*” para, así, encontrar todos los documentos asociados a la raíz del término. De esta manera se incluyen variantes, como singulares y plurales u otras variaciones idiomáticas y se consigue una revisión más exhaustiva.

La búsqueda fue refinada en ambas bases de datos, determinando como criterios de inclusión que las palabras buscadas debían aparecer en el título, en las palabras clave del documento (en el caso de WOS Keyword Plus) o en el resumen y que el tipo de documento sean artículos en inglés o español. En el caso de Scopus se limitó la búsqueda además a la palabra clave exacta "social media" y al estado de publicación final. Cabe mencionar que no se limitaron las fechas de publicación, ya que en ambas bases de datos con las búsquedas efectuadas no se encontraron fechas anteriores al año 2012, excepto un documento de la base de datos Scopus, datado en 2010, que fue eliminado al no cumplir con los criterios de inclusión establecidos. De tal manera se encontraron 139 artículos en WOS y 102 artículos en Scopus.

Una vez obtenidos los datos brutos se usó la herramienta software SciMAT para proceder al análisis de mapeo científico bajo un marco longitudinal. Dicha herramienta permite depurar los datos brutos, usar medidas bibliométricas para estudiar el impacto de cada uno de los elementos y configurar el análisis. Con los mapas de ciencia creados se pueden delimitar las áreas de investigación y analizar la evolución estructural (Cobo, López-Herrera, Herrera-Viedma, \& Herrera, 2012).

En primer lugar, se depuró el corpus de datos. De los 241 artículos importados, quedaron 184 documentos después de haber fusionado los duplicados. Otros 64 documentos se rechazaron por no tener una relación con el objeto de estudio. Los criterios de exclusión se establecieron a partir de la falta de los elementos de referencia (sports tourism, sport event y social media) en los títulos, en las palabras claves o en los resúmenes de los artículos revisados. Además, se implementaron criterios 
de calidad y validación metodológica a juicio del investigador. Por lo tanto, se excluyeron los artículos que solo tienen el "social media" como fuente de recogida de datos, pero cuyo estudio principal no está enfocado en los medios sociales online.

En total se configuró un conjunto de 120 artículos con 412 autores con los cuales se realizó el análisis. En cuanto a las palabras clave, una vez importadas las referencias, se eliminaron las palabras vacías sin significado y se agruparon aquellas de igual significado, identificando así 828 palabras clave.

En el propio análisis con SciMat se dividió el arco cronológico en tres periodos, del 2012 - 2015 (con 17 documentos), del $2016-2018$ (con 43 documentos) y del 2019 - 2021 (con 60 documentos), teniendo un total de 120 documentos en el periodo total del 2012 al 2021 (Nota: Los documentos fueron recogidos el día 23/04/2021, por lo cual no están presentes artículos publicados después de esta fecha).

Como unidad de análisis se eligieron las palabras clave propias de los autores y las de las fuentes, tal y como aparecen indexadas en las bases de datos. Al haber pocos documentos se estableció un mínimo de dos ocurrencias para que aparezca una entidad en el mapa, mientras que la frecuencia mínima de co-ocurrencia se dejó en un mínimo de uno. Se eligió una matrix de co-ocurrencia. Como medida de normalización se usó el índice de equivalencia. El índice de equivalencia es una medida de similitud probabilística y es el más adecuado en los procesos de normalización en la cienciometría (Eck y Waltman, 2009). El algoritmo de agrupamiento usado fue el algoritmo de centros simples, ya que devuelve los clústeres ya etiquetados y está frecuentemente usado en estudios de co-palabras (Cobo et al., 2011). El tamaño máximo de la red se estableció en 12 y el mínimo en tres, para tener una cierta riqueza de construcción semántica.

Los índices de similitud usados para los mapas longitudinales fueron el índice de inclusión para el mapa de evolución y el índice de Jaccard para el mapa de superposición. Se optó por estos dos tipos, ya que el índice de inclusión es más útil a la hora de medir conjuntos similares, mientras que el índice de Jaccard puede ser usado para medir el número de palabras clave compartidas entre subperiodos sucesivos (Cobo et al., 2011). 


\section{RESULTADOS}

Para tener una primera visión general de los artículos publicados en los tres subperiodos analizados se presenta la tabla I. Se verán reflejados el número de publicaciones totales y las citas correspondientes, así como el artículo más citado para cada año con sus autores, la revista en la cual fue publicado y el número de veces que se citó este artículo. Se puede observar que el número total de publicaciones por año estaba creciendo año tras año, aunque el número de citas totales por año estaba decayendo.

Tabla I. Publicaciones y citas por año

\begin{tabular}{|c|c|c|c|c|c|c|}
\hline Año & $\begin{array}{l}\text { Publicaciones } \\
\text { totales por } \\
\text { año }\end{array}$ & $\begin{array}{l}\text { Citas } \\
\text { totales } \\
\text { por año }\end{array}$ & Artículo más citado & Autores & $\begin{array}{l}\text { № } \\
\text { de } \\
\text { citas }\end{array}$ & Fuente \\
\hline 2012 & 3 & 34 & $\begin{array}{l}\text { The Role of Belief in Making a Difference in Enhancing Attachment } \\
\text { to a Charity Sport Event }\end{array}$ & $\begin{array}{l}\text { Filo, K.; Groza M.; Fairley, } \\
\text { S. }\end{array}$ & 21 & $\begin{array}{l}\text { Journal of } \\
\text { Nonprofit \& Public } \\
\text { Sector Marketing }\end{array}$ \\
\hline 2013 & 1 & 16 & Sentiment Identification in Football-Specific Tweets & Aloufi, S.; Saddik, A.E. & 16 & IEEE Access \\
\hline 2014 & 5 & 96 & Digital cultures, acceleration and mega sporting event narratives & McGillivray, D. & 30 & Leisure Studies \\
\hline 2015 & 8 & 154 & $\begin{array}{l}\text { How social media engagement leads to sports channel loyalty: } \\
\text { Mediating roles of social presence and channel commitment }\end{array}$ & $\begin{array}{l}\text { Lim, J. S.; Hwang, Y. C.; } \\
\text { Kim, S.; Biocca, F. A. }\end{array}$ & 79 & $\begin{array}{l}\text { Computers in } \\
\text { Human Behavior }\end{array}$ \\
\hline 2016 & 13 & 129 & $\begin{array}{l}\text { Exploring PERMA in spectator sport: Applying positive psychology to } \\
\text { examine the individual-level benefits of sport consumption }\end{array}$ & $\begin{array}{l}\text { Filo, K.; Doyle, J. P.; Lock, } \\
\text { D.; Funk, D. C.; } \\
\text { McDonald, H. }\end{array}$ & 36 & $\begin{array}{l}\text { Sport } \\
\text { Management } \\
\text { Review }\end{array}$ \\
\hline 2017 & 10 & 77 & $\begin{array}{l}\text { To post or not to post: social media sharing and sporting event } \\
\text { performance }\end{array}$ & $\begin{array}{l}\text { Prado-Gascó, V.; Calabuig } \\
\text { Moreno, F., Añó Sanz, V.; } \\
\text { Núñez-Pomar, J.; Crespo } \\
\text { Hervàs, J. }\end{array}$ & 14 & $\begin{array}{l}\text { Psychology \& } \\
\text { Marketing }\end{array}$ \\
\hline 2018 & 20 & 131 & $\begin{array}{l}\text { Sports fan experience: Electronic word-of-mouth in ephemeral } \\
\text { social media }\end{array}$ & $\begin{array}{l}\text { Wakefield, L. T.; Bennett, } \\
\text { G. }\end{array}$ & 21 & $\begin{array}{l}\text { Sport } \\
\text { Management } \\
\text { Review }\end{array}$ \\
\hline 2019 & 16 & 53 & $\begin{array}{l}\text { Twitter, Team GB and the Australian Olympic Team: representations } \\
\text { of gender in social media spaces }\end{array}$ & Litchfield, C.; Kavanagh, E. & 10 & Sport in Society \\
\hline 2020 & 36 & 44 & $\begin{array}{l}\text { Social media and inspiring physical activity during COVID-19 and } \\
\text { beyond }\end{array}$ & Hayes, $\mathrm{M}$. & 6 & $\begin{array}{l}\text { Managing Sport } \\
\text { and Leisure }\end{array}$ \\
\hline \multirow{2}{*}{2021} & \multirow[b]{2}{*}{8} & \multirow{2}{*}{11} & $\begin{array}{l}\text { Athletes as ambush marketers? An examination of Rule } 40 \text { and } \\
\text { athletes' social media use during the } 2016 \text { Rio Olympic Games }\end{array}$ & $\begin{array}{l}\text { Geurin, A. N.; McNary, E. } \\
\text { L. }\end{array}$ & \multirow[b]{2}{*}{3} & $\begin{array}{l}\text { European Sport } \\
\text { Management } \\
\text { Quarterly }\end{array}$ \\
\hline & & & $\begin{array}{l}\text { Life is more important than football: Comparative analysis of } \\
\text { Tweets and Facebook comments regarding the cancellation of the } \\
2015 \text { African Cup of Nations in Morocco }\end{array}$ & $\begin{array}{l}\text { Moreau, N.; Roy, M.; } \\
\text { Wilson, A.; Atlani Duault, } \\
\text { L. }\end{array}$ & & $\begin{array}{l}\text { International } \\
\text { Review for the } \\
\text { Sociology of Sport }\end{array}$ \\
\hline Total & 120 & 745 & & & & \\
\hline
\end{tabular}

En el mapa de superposición (figura 1) se pueden ver, siguiendo el modelo de Price y Gürsey (1975), la evolución de las palabras clave y las medidas de estabilidad entre los tres periodos consecutivos. Los círculos representan los periodos y el número dentro del círculo son las palabras clave asociadas a este periodo. Las flechas superiores salientes representan las palabras clave que no pasan de un periodo a otro, 
mientras que las flechas superiores entrantes representan las nuevas palabras clave en el siguiente periodo. Las flechas horizontales muestran el número de las palabras clave que pasan de un periodo al siguiente, siendo el número en paréntesis el índice de similitud entre ellas.



Figura 1. Mapa de superposición

En el primer periodo (A) se identificaron 130 palabras clave, 52 de ellas se traspasaron al segundo periodo (B), en el cual aparecieron 276 nuevas palabras clave. Del total de las 328 palabras clave del periodo B, 115 pasaron al último periodo analizado (C) que incluyó 311 nuevas palabras clave, teniendo un total de 426.

El número total de palabras clave aumentó en gran medida, habiendo más del triple del número inicial en el último periodo. De la misma manera se ve que el índice de similitud aumentó del 0,13 al 0,18 entre los subperiodos, lo cual significa que hubo una ligera consolidación en la terminología del campo. No obstante, el número de palabras transversales fue alto, ya que hubo un gran número de palabras que sólo estuvo presente en uno de los subperiodos y que no traspasó al siguiente. El número de palabras claves nuevas añadidas en el siguiente periodo superó en gran número las palabras clave que se importaron del subperiodo anterior. 


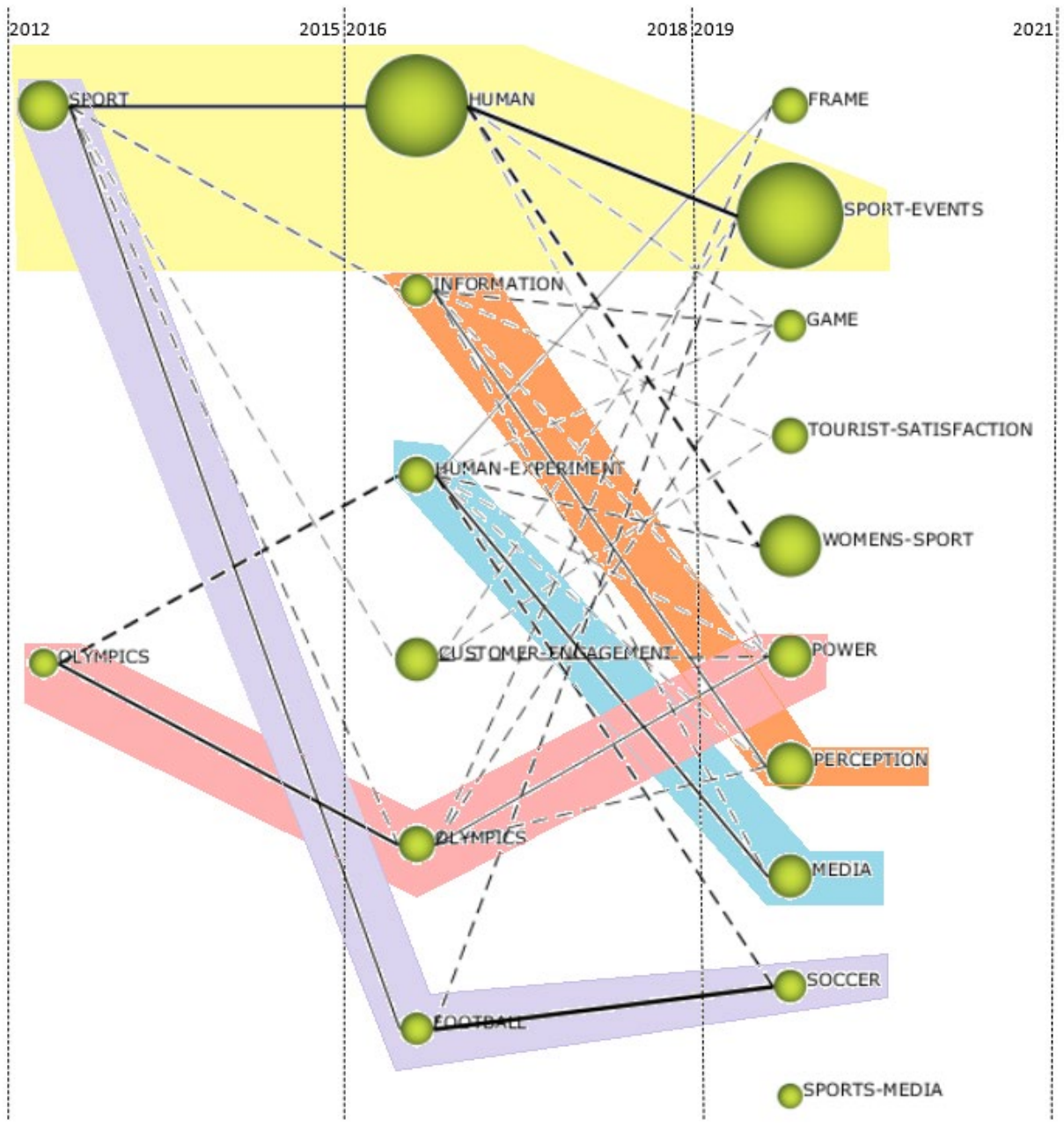

Figura 2. Mapa de superposición

Tras haber visto la evolución de las palabras clave, se muestra la evolución temática de las áreas detectadas (figura 2). Como anteriormente se ha explicado, se dividió el análisis en tres periodos. Las líneas sólidas en la figura representan vínculos temáticos y las líneas trazadas conectan temas que comparten elementos que no son el nombre del tema. El grosor de la línea es proporcional al índice de inclusión y el tamaño de las esferas representa el número de documentos pertenecientes al tema. 
En el primer periodo sólo se detectaron dos áreas temáticas: "sport" y "olympics". Mientras "olympics" se quedaba presente en el segundo periodo, "sport" evolucionó hacia el vínculo con "human" y posteriormente a "sport-events" y por otro lado a "football" y "soccer".

En el periodo desde el año 2016 al 2018 se identificaron seis áreas temáticas. Tanto "información" como "human-experiment" son temas que estuvieron conectados con "sport" y "olympics" respectivamente, aunque compartieron palabras claves diferentes a sus respectivos nombres. El área temática "information" en el último periodo se vio evolucionando hacia el término "perception" y "human-experiment" se vio conectado con "media".

En el último periodo del año 2019 al año 2021 se identificaron diez áreas temáticas. Apareció una nueva área temática: "sports-media", la única que no presenta ninguna conexión con los demás temas.

Tabla II. Rendimiento de las áreas temáticas

\begin{tabular}{llcccc}
\hline Periodo & Nombre & Documentos & Índice $\mathbf{h}$ & $\begin{array}{c}\text { Promedio } \\
\text { de citas }\end{array}$ & $\begin{array}{c}\text { Suma de } \\
\text { citas }\end{array}$ \\
\hline $2012-2015$ & SPORT & 9 & 7 & 24.11 & 217 \\
$2012-2015$ & OLYMPICS & 3 & 2 & 10.67 & 32 \\
\hline $2016-2018$ & HUMAN & 23 & 8 & 6.7 & 154 \\
$2016-2018$ & INFORMATION & 4 & 4 & 10 & 40 \\
$2016-2018$ & HUMAN-EXPERIMENT & 5 & 4 & 11.6 & 58 \\
$2016-2018$ & CUSTOMER- & 7 & 5 & 9 & 63 \\
$2016-2018$ & OLYMPICS & 5 & 4 & 14.2 & 71 \\
$2016-2018$ & FOOTBALL & 4 & 3 & 9.25 & 37 \\
\hline $2019-2021$ & FRAME & 5 & 2 & 3 & 15 \\
$2019-2021$ & SPORT-EVENTS & 24 & 5 & 2.71 & 65 \\
$2019-2021$ & GAME & 4 & 3 & 3.75 & 15 \\
$2019-2021$ & TOURIST-SATISFACTION & 5 & 3 & 3.8 & 19 \\
$2019-2021$ & WOMENS-SPORT & 12 & 3 & 2.08 & 25 \\
$2019-2021$ & POWER & 7 & 3 & 2.71 & 19 \\
$2019-2021$ & PERCEPTION & 8 & 3 & 2 & 16 \\
$2019-2021$ & MEDIA & 7 & 2 & 1.14 & 8 \\
$2019-2021$ & SOCCER & 4 & 2 & 2.5 & 10 \\
$2019-2021$ & SPORTS-MEDIA & 2 & 1 & 2 & 4 \\
\hline
\end{tabular}

Mediante los diagramas estratégicos se visualizan los temas en cuatro cuadrantes, según las medidas de análisis de redes temáticas de Callon et al. (1991): la centralidad y la densidad. 
La primera de ellas, la centralidad, representada en el eje de abscisas, mide el grado de fuerza de enlaces externos de un tema con otros. Por lo cual puede ser interpretado como medida de la importancia de un tema en el desarrollo general del campo científico analizado. En cambio, la densidad, representada en el eje de ordenadas, mide la fuerza de los enlaces internas de entre todas las palabras clave que describen el tema y puede ser interpretada como medida del nivel de desarrollo del tema (Cobo et al., 2011).

Los cuatro cuadrantes representan cada uno una categoría distinta:

Cuadrante 1 (superior derecho): Temas motores

Tienen una alta centralidad y densidad. Estos temas corresponden a temas desarrollados y que tienen importancia en la construcción del campo científico.

Cuadrante 2 (superior izquierdo): Temas periféricos, temas aislados o muy desarrollados

Representan temas bien especializados, es decir desarrollados internamente, pero aislados del resto de los temas, por lo cual tienen una importancia marginal en el desarrollo del campo científico.

Cuadrante 3 (inferior izquierdo): Temas emergentes o en desaparición

Al presentar tanto una baja centralidad como una baja densidad, estos temas o son temas en declive o temas emergentes que aún están poco desarrollados.

Cuadrante 4 (inferior derecho): Temas básicos y transversales

Estos temas genéricos y transversales tienen importancia para el campo científico, pero se encuentran poco desarrollados. 




Figura 3. Diagrama estratégico periodo A (2012 - 2015)



Figura 4. Diagrama estratégico periodo B (2016 - 2018) 


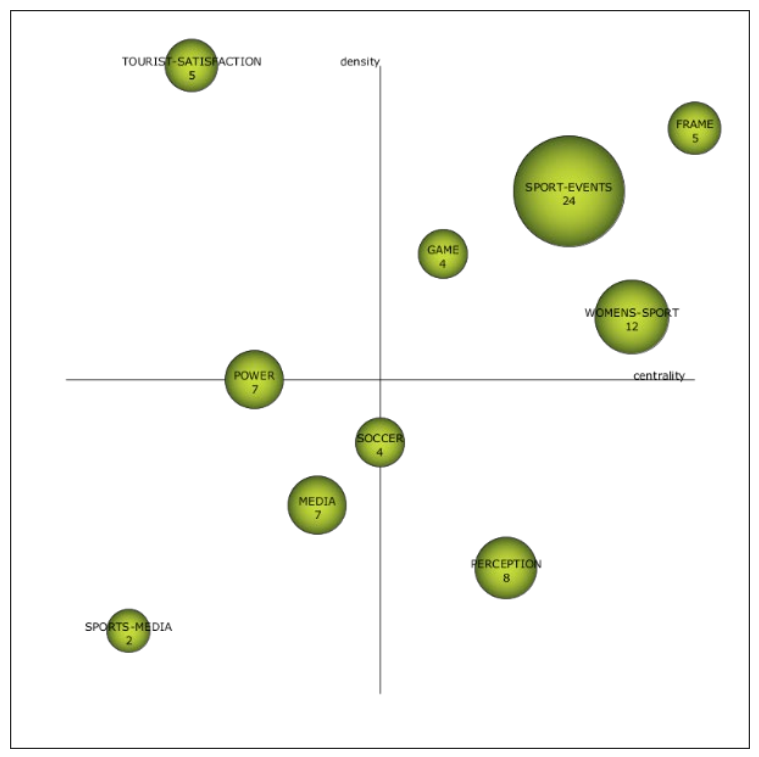

Figura 5. Diagrama estratégico periodo C (2019 - 2021)

En las figuras 3, 4 y 5 se visualiza la evolución de los temas principales en los tres periodos anteriormente establecidos. En los círculos se incluye el número de publicaciones de cada tema. A través de estas representaciones gráficas se analizan los temas más relevantes de cada periodo. Además, en la tabla II se detallan los índices de rendimiento de los temas anteriormente identificados.

En el periodo A, al tratarse de un campo relativamente nuevo, todavía existen pocas publicaciones y sólo se identifican dos temas. Por un lado "olympics" que se centra en medio de los dos ejes, obtiene una moderada tasa de impacto. En cambio "sport" muestra una alta densidad y centralidad y por lo tanto es el tema motor en este periodo. Asimismo, destaca por el índice h (7) y el impacto más alto en el promedio de citas (24.11) y en la suma de citas (217), de todas las áreas identificadas en todos los periodos.

En el segundo periodo (2016 - 2018) los temas "human" y "humanexperiment" se categorizan como temas motores, teniendo "human" el índice $\mathrm{h}$ de 8 , el más alto de todos los temas identificados. "Humanexperiement" tiene el segundo promedio de citas más elevado $(11,6)$ después de "olympics" $(14,2)$ en el periodo $\mathrm{B}$, mientras que el promedio de citas de "human" es bajo $(6,7)$. 
"Olympics" aumenta desde el primer periodo al segundo en todos los indicadores. El hecho de que no está presente en el último periodo puede verse afectado en gran medida por la cancelación de los Juegos Olímpicos de Tokio en el año 2020 y la subsiguiente falta de datos para investigaciones en ellos.

En cuanto a su posicionamiento en el cuadrante, muestra una densidad más baja y la misma centralidad que en el primer periodo. Aunque según el aumento sufrido en todos sus indicadores, que muestra su tendencia de crecimiento, la tasa de impacto en el desarrollo del campo científico sigue baja.

En el último periodo (2019 - 2021) se encuentran diez temas. A la vista del rendimiento de estas áreas temáticas del campo, cabe señalar que las más destacables son "sport-events", que tiene el mayor número de documentos, la mayor suma de citas e índice $h$, seguido por "womenssport", referente al número de documentos y la suma de citas, y "touristsatisfaction", con el índice $h$ más elevado (3.8) en este periodo. Este último, aunque destaca por el índice $h$, juega un rol marginal para el desarrollo de este campo científico, ya que presenta una centralidad baja y una muy alta densidad.

Se observan cuatro temas motores: "sport-events", "womens-sports", "frame" y "game", siendo los dos primeros los más destacables en los números de indicadores.

"Percepción" es un tema básico, que tiene importancia para el campo científico, pero que se encuentra en un estado aún no bien desarrollado.

Por último, cabe destacar "sports-media", por su baja centralidad y baja densidad. Se encuentra poco desarrollado y supone un tema emergente en este campo. 


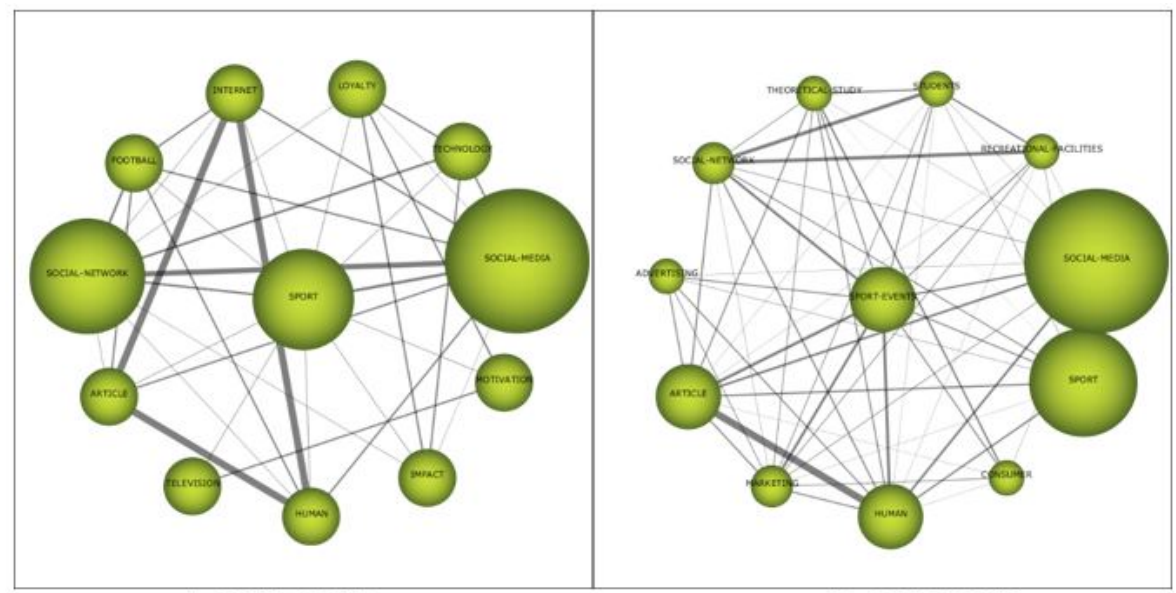

A: $2012-2015$

B: $2016-2018$



C: $2019-2021$

Figura 2. Clústers principales

Se ha visto anteriormente en los análisis de la evolución temática y del rendimiento de las áreas temáticas que no están presentes las palabras clave relacionadas con los medios sociales, tales como "social media" o "social network". Aun así, al ver los clústers de los principales temas motores de los tres periodos analizados ("sport", "human" y "sportevent"), cabe destacar que cada uno de ellos está estrechamente relacionado con "social-media" y con "social-network". 
En el segundo periodo se puede observar asimismo tanto la relación entre "social-media" con "sport" y "twitter", como entre "socialnetwork" con "sport-events".

El tema emergente "sports-media" se encuentra relacionado con "digital-media", de los cuales forman parte los medios sociales.

\section{DISCUSIÓN Y CONCLUSIONES}

A la vista de la evolución temática de las áreas detectadas, se observa generalmente poca cohesión sólida entre los temas identificados. No obstante, tampoco se encuentran vacíos y las áreas están evolucionando. Al tratarse de un campo relativamente nuevo, los temas se encuentran en fase de desarrollo. De los dos temas del primer periodo se evoluciona a seis en el segundo periodo y a diez en el último periodo.

Como síntesis general, el presente estudio ha aplicado un análisis bibliométrico para identificar y visualizar el estado actual de la investigación en el campo del turismo deportivo y las redes sociales. Tanto el turismo deportivo como las redes sociales han sido analizados en sus respectivos campos. A pesar de ello, las investigaciones de estos dos campos juntos se encuentran en una fase de desarrollo y crecimiento, y todavía no se observa una consolidación en el área. El número de publicaciones tiene una tendencia creciente desde el año 2012 hasta la actualidad, mientras que el total de citas por año desciende. En cuanto a la superposición de las palabras clave, ésta aumenta drásticamente desde el primer periodo y se observa una ligera consolidación en el último periodo. El número de temas identificados aumenta a lo largo de los subperiodos. Los principales temas se encuentran poco conectados entre ellos, pero sin vacíos, lo cual muestra el estado inicial de evolución hacia un campo desarrollado.

Los temas motores son "sport" (periodo A), "human", "humanexperiment" (periodo B), "sport-events", "woman-sports", "frame" y "game" (periodo $\mathrm{C}$ ), teniendo que recalcar en este último periodo "sports-media" como tema emergente. Si bien no se identifican "socialmedia" o "social-network" como áreas temáticas, cabe destacar que están fuertemente relacionados con los principales temas motores de los tres periodos analizados, "sport", "human" y "sport-event".

Los resultados obtenidos en este estudio cuentan con la limitación que supone el uso de unas determinadas fuentes de información junto con la definición de un perfil concreto de búsqueda. Futuras investigaciones 
pueden incluir otras bases de datos y una vez que haya una cierta consolidación del campo, analizar la evolución temática en un periodo más prolongado.

\section{BibLIOGRAFÍA}

Abeza, G., O'Reilly, N., \& Reid, I. (2013). Relationship Marketing and Social Media in Sport. International Journal of Sport Communication, 6(2), 120142. DOI: https://doi.org/10.1123/ijsc.6.2.120

Anagnostopoulos, C., Parganas, P., Chadwick, S., \& Fenton, A. (2018). Branding in pictures: Using Instagram as a brand management tool in professional team sport organisations. European Sport Management Quarterly, 18(4), 413-438.

DOI: https://doi.org/10.1080/16184742.2017.1410202

Baccarella, C. V., Wagner, T. F., Kietzmann, J. H., \& McCarthy, I. P. (2018). Social media? It's serious! Understanding the dark side of social media. European Management Journal, 36(4), 431-438. DOI: https://doi.org/10.1016/j.emj.2018.07.002

Callon, M., Courtial, J., \& Laville, F. (1991). Co-word analysis as a tool for describing the network of interactions between basic and technological research: The case of polymer chemsitry. Scientometrics, 22(1), 155-205. DOI: https://doi.org/10.1007/bf02019280

Cobo, M. J., López-Herrera, A. G., Herrera-Viedma, E., \& Herrera, F. (2011). An approach for detecting, quantifying, and visualizing the evolution of a research field: A practical application to the Fuzzy Sets Theory field. Journal of Informetrics, 5(1), 146-166.

DOI: https://doi.org/10.1016/j.joi.2010.10.002

Cobo, M. J., López-Herrera, A. G., Herrera-Viedma, E., \& Herrera, F. (2012). SciMAT: A new science mapping analysis software tool. Journal of the American Society for Information Science and Technology, 63(8), 16091630. DOI: https://doi.org/10.1002/asi.22688

Cropanzano, R. (2009). Writing Nonempirical Articles for Journal of Management: General Thoughts and Suggestions. Journal of Management, 35(6), 1304-1311. DOI: https://doi.org/10.1177/0149206309344118 
De Knop, P. (1990). Sport for all and active tourism. World Leisure \& Recreation, 32(3), 30-36.

Eck, N. J. van, \& Waltman, L. (2009). How to normalize cooccurrence data? An analysis of some well-known similarity measures. Journal of the American Society for Information Science and Technology, 60(8), 1635-1651. DOI: https://doi.org/10.1002/asi.21075

Filo, K., Lock, D., \& Karg, A. (2015). Sport and social media research: A review. Sport Management Review, 18(2), 166-181. DOI: https://doi.org/10.1016/j.smr.2014.11.001

Fundación Española para la Ciencia y la Tecnología. (s. f.). Recursos Científicos | Productos contratados. Recuperado el 3 de mayo de 2021, de https://www.recursoscientificos.fecyt.es/licencias/productos-contratado

Gammon, S., \& Robinson, T. (2003). Sport and Tourism: A Conceptual Framework. Journal of Sport \& Tourism, 8:1, 21-26. DOI: https://doi.org/10.1080/14775080306236

Gibson, H. (1998). Sport Tourism: A Critical Analysis of Research. Sport Management Review, 33.

Girginov, V., Taks, M., Boucher, B., Martyn, S., Holman, M., \& Dixon, J. (2009). Canadian National Sport Organizations' Use of the Web for Relationship Marketing in Promoting Sport Participation. International Journal of Sport Communication, 2(2), 164-184. DOI: https://doi.org/10.1123/ijsc.2.2.164

Glyptis, S. (1982). Sport and tourism in Western Europe. British Travel Educational Trust.

González, J. S. (2012). Deporte y social media: El caso de la Primera División del fútbol español. Historia y Comunicación Social, 17, 217-230. DOI: https://doi.org/10.5209/rev_HICS.2012.v17.40607

Grönroos, C. (2004). The relationship marketing process: Communication, interaction, dialogue, value. Journal of Business \& Industrial Marketing, 19(2), 99-113. DOI: https://doi.org/10.1108/08858620410523981

Hayes, M., Filo, K., Riot, C., \& Geurin, A. N. (2019). Athlete Perceptions of Social Media Benefits and Challenges During Major Sport Events. 
International Journal of Sport Communication, 12(4), 449-481. DOI: https://doi.org/10.1123/ijsc.2019-0026

Kaplan, A. M., \& Haenlein, M. (2010). Users of the world, unite! The challenges and opportunities of Social Media. Business Horizons, 53(1), 59-68. DOI: https://doi.org/10.1016/j.bushor.2009.09.003

Kitchenham, B. (2004). Procedures for performing systematic reviews. Keele, UK, Keele University, 33(2004), 1-26.

López-Carril, S., Villamón, M., \& Sanz, V. A. (2019). Conceptualización de los medios sociales: Oportunidades para la gestión del deporte (Conceptualisation of Social Media: opportunities for Sport Management). Retos: nuevas tendencias en educación física, deporte y recreación, (36), 578-583.

Meng, M. D., Stavros, C., \& Westberg, K. (2015). Engaging fans through social media: Implications for team identification. Sport, Business and Management: An International Journal, 5(3), 199-217. DOI: https://doi.org/10.1108/SBM-06-2013-0013

Noyons, E. C. M., Moed, H. F., \& Luwel, M. (1999). Combining mapping and citation analysis for evaluative bibliometric purposes: A bibliometric study. Journal of the American Society for Information Science, 50(2), 115-131. https://doi.org/10.1002/(SICI)1097-4571(1999)50:2<115::AID$\mathrm{ASI} 3>3.0 . \mathrm{CO} ; 2-\mathrm{J}$

Price, D., \& Gürsey, S. (1975). Studies in Scientometrics I Transience and Continuance in Scientific Authorship.

DOI: https://doi.org/10.18225/CI.INF..V4I1.64

Pritchard, A. (1969). Statistical Bibliography or Bibliometrics? Journal of Documentation, 25, 348-349.

Small, H. (1973). Co-citation in the scientific literature: A new measure of the relationship between two documents. Journal of the American Society for Information Science, 24(4), 265-269.

DOI: https://doi.org/10.1002/asi.4630240406

Stavros, C., Meng, M. D., Westberg, K., \& Farrelly, F. (2014). Understanding fan motivation for interacting on social media. Sport Management Review, 17(4), 455-469. DOI: https://doi.org/10.1016/j.smr.2013.11.004 
Sutton, W. A., McDonald, M. A., Milne, G. R., \& Cimperman, J. (1997). Creating and fostering fan identification in professional sports. Sport marketing quarterly, 6, 15-22.

Tourism Economics. (2020). Sports Tourism: State of the Industry Report (2019). Recuperado el 14 de mayo de 2021, de https://www.sportseta.org/portals/sportscommissions/Documents/Reports/ TourismEconomics $\% 20-\% 20$ Sports $\% 20$ ETA $\% 20$ SOTI $\% 20$ \%20FINAL_82620.pdf

Weed, M. (2005). Sports Tourism Theory and Method-Concepts, Issues and Epistemologies. European Sport Management Quarterly, 5(3), 229-242. DOI: https://doi.org/10.1080/16184740500190587

Weed, M., \& Bull, C. (2009). Sports Tourism: Participants, Policy \& Providers (2. ${ }^{a}$ ed.). Oxford: Elsevier.

World Tourism Organization. (s. f.). Sports Tourism. Recuperado el 23 de enero de 2021, de https://www.unwto.org/sport-tourism 